\title{
Disorder-driven superconductor-normal metal phase transition in quasi-one-dimensional organic conductors
}

\author{
E. Nakhmedov ${ }^{1,2}$ and R. Oppermann ${ }^{1,3}$ \\ ${ }^{1}$ Institut für Theoretische Physik, Universität Würzburg, D-97074 Würzburg, Germany \\ ${ }^{2}$ Institute of Physics, Azerbaijan National Academy of Sciences, H. Cavid str. 33, AZ1143 Baku, Azerbaijan \\ ${ }^{3}$ Institut de Physique Théorique, CEA Saclay, Orme des Merisiers, F-91191 Gif-sur-Yvette, France
}

(Dated: November 11, 2018)

\begin{abstract}
Effects of non-magnetic disorder on the critical temperature $T_{c}$ and on diamagnetism of quasione-dimensional superconductors are reported. The energy of Josephson-coupling between wires is considered to be random, which is typical for dirty organic superconductors. We show that this randomness destroys phase coherence between wires and that $T_{c}$ vanishes discontinuously at a critical disorder-strength. The parallel and transverse components of the penetration-depth are evaluated. They diverge at different critical temperatures $T_{c}^{(1)}$ and $T_{c}$, which correspond to pairbreaking and phase-coherence breaking respectively. The interplay between disorder and quantum phase fluctuations is shown to result in quantum critical behavior at $T=0$, which manifests itself as a superconducting-normal metal phase transition of first-order at a critical disorder strength.
\end{abstract}

PACS numbers: 74.78.-w, 74.62.-c, 74.70.Kn, 74.50.+r

\section{INTRODUCTION}

Although more than a quarter of a century has elapsed since the discovery of superconductivity in the quasi-one-dimensional (quasi-1D) organic charge-transfer (Bechgaard) salts of $(T M T S F)_{2} X$-type (where TMTSF stands for tetramethyltetraselenofulvalinium and $X=$ $\mathrm{PF}_{6}, \mathrm{ClO}_{4}, \mathrm{NO}_{3}$ being a strong electron acceptor or anion $)^{1,2}$, many distinct properties of this material still remain a matter of debate. Among those one may mention the pairing symmetry, the remarkable sensitivity of the critical temperature to irradiation ${ }^{3,4}$, large upper critical field $H_{c 2}$ etcetera (see, also Ref.5). The irradiation destroys anion order, introducing thus nonmagnetic damage into system that led Abrikosov to suggest the possibility of triplet pairing ${ }^{6}$ in the organic salts. Nevertheless, the experimental evidence ${ }^{7}$ that the spinsusceptibility decreases at low magnetic fields in the superconducting (SC) state of the (TMTSF $)_{2} \mathrm{ClO}_{4}$ organic conductor, disfavors the triplet pairing mechanism and supports spin-singlet pairing.

Low temperature properties of organic superconductors are known to be very sensitive to disorder. Alloying with anions, $x$-ray irradiation, or cooling rate controlled anion reorientation introduces non-magnetic randomness into the system, however leaving unchanged, to a large extent, the backbone structure and the unit cell of the organic superconductors. There is a common agreement that disorder, when introduced by means of these experimental methods, must be characterized as non-magnetic. Yet, it was shown ${ }^{3,8-10}$ to suppress the $\mathrm{SC}$ phase. It is worth noting that the methods of generating non-magnetic disorder in layered organic superconductors are similar (see, e.g. Ref.11) to those in quasi-1D systems, and, therefore a similar mechanism of SC state suppression in these two classes of materials may exist.

Studies of disorder effects on the superconducting phase have a long history. The superconducting transi- tion temperature $T_{c}$ for $s$-wave pairing has been shown to be insensitive to the scattering rate on non-magnetic impurities, which is known as the Anderson theorem ${ }^{12}$. In contrast to non-magnetic impurities, paramagnetic impurities break time-reversal symmetry of the $s$-pairing, and suppress the SC-phase ${ }^{13}$ at some critical concentration of the impurities. Correlation between the paramagnetic impurities via the Ruderman-Kittel-KasuyaYosida (RKKY) interaction yields a spin-glass phase below $T=T_{g}$, which was shown ${ }^{14}$ to shift the superconducting transition point towards higher temperatures. The Anderson theorem is not applicable to unconventional superconductors with $d$-wave pairing symmetry ${ }^{15}$. A small concentration of non-magnetic impurities may destroy $d$-wave pairing, producing a finite lifetime for quasi-particles near the nodes in the gap ${ }^{16-18}$.

Inter-chain (inter-layer) pairings as well as intra-chain (intra-layer) pairings, occurring at different local critical temperatures in quasi-1D (quasi-2D) systems, yield also an inhomogeneous nodal order parameter ${ }^{19,20}$, which affect considerably the upper critical magnetic field.

Suppression of superconductivity in the presence of non-magnetic impurities can in general be realized by destroying either the modulus or the phase coherence of the order parameter. Interplay between superconductivity and Anderson localization in a strongly disordered superconductor was shown ${ }^{21-30}$ to result in spatial inhomogeneity of the order parameter. Diffusive scattering of particles in the random field of non-magnetic impurities enhances Coulomb repulsion ${ }^{31,32}$, and consequently, reduces the amplitude of the order parameter. Mesoscopic fluctuations in a superconducting thin film were also shown ${ }^{33}$ to yield a spatial inhomogeneity of the order parameter.

Effects of order parameter phase fluctuations on the superconducting transition temperature $T_{c}$ have been studied in Refs.34-38. It is well known that there is no SC phase transition in one-dimensional (1D) and two- 
dimensional (2D) systems ${ }^{39}$, since strong fluctuations of the order parameter phase destroy off-diagonal longrange order (ODLRO) in a single $\mathrm{SC}$ wire and in an isolated SC film. Even a small interchain-coupling in clean quasi-1D superconductors restores however ODLRO together with a finite transition temperature. The suppression of $T_{c}$ by strong phase fluctuations in clean quasi$1 \mathrm{D}$ superconductors was analyzed in Ref.34,35. Classifying superconductors with small stiffness as bad metals, Emery and Kivelson have evaluated ${ }^{37}$ a critical temperature of phase ordering by formally dividing a clean bulk superconductor into small regions with well defined phase, and have shown considerable suppression of an SC-phase by phase fluctuations. Effects of disorder on phase fluctuations were however not considered in all of these papers.

Distinct structural peculiarities of quasi-1D organic superconductors demand a special theory, which should take into account non-magnetic randomness as well as phase fluctuations. Indeed, the high purity of the organic superconductor backbone, even in an overall dirty limit, excludes spatial inhomogeneity of the order parameter modulus along the SC-wires. This renders inapplicable the above-mentioned theories for pair breaking.

In contrast to these previous activities we study in this article a disorder-driven superconductor-normal metal phase transitions due to the competition of nonmagnetic randomness and phase-fluctuations in quasi-1D superconductors. We consider weakly linked quasi-onedimensional superconductors with random Josephsoncouplings between pure one-dimensional (1D) SC wires. Singlet pairing is assumed within the wires. Therefore, we assume that non-magnetic randomness does not affect the order parameter amplitude.

We demonstrate in this article that

(i) non-magnetic randomness in the Josephsoncoupling destroys correlation of the phases between different chains in quasi-1D superconductors even in the classical phase fluctuation regime,

(ii) non-magnetic randomness yields quantum critical behavior in addition. A superconducting normal-metal phase transition occurs at $T=0$ increasing the strength of disorder, and

(iii) a suppression of the SC phase occurs discontinuously as well both the classical and the quantum phase fluctuation regimes as a first-order phase transition when the disorder-strength reaches a critical value. We derive parallel and perpendicular components of the penetration depth, $\lambda_{\|}$and $\lambda_{\perp}$, and show that they diverge at different critical temperatures $T_{c}^{(1)}$ and $T_{c}$, which correspond to pair-breaking in the wires and to phase coherence breaking between the SC wires, respectively.

The paper is organized as follows: In Sec. II we study the interplay of randomness in the Josephson energy with phase fluctuations inside the classical regime. Although an arbitrary small interchain coupling in a clean quasi-1D superconductor stabilizes the ODLRO giving a finite transition temperature $T_{c}$, we show in this Sec- tion that non-magnetic disorder in the Josephson energy suppresses $T_{c}$ discontinuously when the disorder-strength reaches a critical value. In Sec. III, we investigate effects of randomness on the transverse rigidity and on $T_{c}$ in the quantum fluctuations regime. We show in this Section that a suppression of the SC phase is managed by two parameters characterizing the disorder-strength and a dynamical charging parameter $\alpha$ in the system. The quantum criticality at $T=0$ and the phase transition at finite temperature are described separately. The transverse rigidity in the field of the phases is shown to vanish discontinuously. The jump at the breakdown point decreases monotonically with increasing $\alpha$ in the interval of $0 \leq \alpha<1$, and vanishes for $\alpha=1$. The breakdown point is pushed towards higher values of the disorderstrength as $\alpha$ increases. We show that, under a change of the disorder-strength, the critical temperature evolves similarly as the transverse rigidity at $T=0$. The analysis of diamagnetism in this Section reveals completely different behavior for parallel and perpendicular components of the penetration depth. The penetration depth for a magnetic field parallel to the SC-wires is shown to be defined by the phase-phase correlator between two neighboring wires, which behaves non-linearly in temperature and reveals a discontinuous dependence on disorder; by contrast the perpendicular penetration depth does not depend on disorder and shows a conventional temperature dependence. In Sec. V we summarize results obtained in the paper. The explicit evaluation of the phase-phase correlator is given in the Appendix.

\section{CLASSICAL PHASE FLUCTUATION REGIME}

A quasi-1D superconductor is modeled as a system of one-dimensional wires, which are placed regularly and parallel to each other, forming for example a square lattice in the cross-section. Weak tunneling between the chains results in an open Fermi surface for the normal metallic state, and yields also the Josephson-coupling between nearest-neighboring chains in the superconducting state and strong anisotropy in kinetic properties.

The free energy functional of a quasi-1D superconductor, which is weakly linked with Josephson-coupling energy $E_{\mathbf{j}, \mathbf{j}+\mathbf{g}}$ between nearest-neighbor chains, can be written in the presence of the magnetic field $\mathbf{B}$ as

$$
\begin{aligned}
& F_{s t}=N_{s}^{(1)}(T) \xi_{\|} \sum_{\mathbf{j}} \int d z\left\{\frac{\hbar^{2}}{8 m_{\|} \xi_{\|}^{2}}\left(\frac{\partial \varphi_{\mathbf{j}}}{\partial z}-\frac{2 e \xi_{\|}}{\hbar c} A_{z}\right)^{2}+\right. \\
& \sum_{\mathbf{g}= \pm 1} E_{\mathbf{j}, \mathbf{j}+\mathbf{g}}\left[1-\cos \left(\varphi_{\mathbf{j}}-\varphi_{\mathbf{j}+\mathbf{g}}+\frac{2 e \xi_{\|}}{\hbar c} \int_{\mathbf{j}}^{\mathbf{j}+\mathbf{g}} \mathbf{A}_{\perp} d \mathbf{r}_{\perp}\right)\right] \\
&\left.\quad+\xi_{\|} a_{\perp}^{2} \frac{\left(\mathbf{B}(\mathbf{r})-\mathbf{B}_{e x t}\right)^{2}}{8 \pi}\right\},
\end{aligned}
$$

where $\varphi_{\mathbf{j}}(z)$ denotes the phase of the order parameter $\Delta_{\mathbf{j}}(z)=\left|\Delta_{\mathbf{j}}\right| \exp \left(i \varphi_{\mathbf{j}}(z)\right)$ at a point with dimensionless 
coordinates $\mathbf{r}=\left\{\mathbf{j}=\left\{j_{x}, j_{y}\right\}, z\right\}, \mathbf{A}=\left\{\mathbf{A}_{\perp}, A_{z}\right\}$ is the vector-potential, and $N_{s}^{(1)}$ the linear density of SC electrons with $N_{s}^{(1)}(T)=N_{s}^{(1)}(0) \equiv N_{N}^{(1)} \simeq \frac{p_{F}}{\hbar}$ at $T \geq T_{c}^{(1)}$, and $N_{s}^{(1)}(T)=N_{s}^{(1)}(0) \tau(T)$ with $\tau(T)=\frac{T_{c}^{(1)}-T}{T_{c}^{(1)}}$ at $T \leq$ $T_{c}^{(1)}$. Dimensionless coordinates $\mathbf{r}=\{\mathbf{j}, z\}$ are introduced on the scale of the longitudinal $\xi_{\|}=\frac{\hbar^{2} N_{s}^{(1)}(0)}{4 m_{\|} T_{c}^{(1)}}$ and the transverse $\xi_{\perp} \sim a_{\perp}$ components of the coherence length, so $\mathbf{r}=\left\{r_{\|}=\left\{j_{x} a_{\perp}, j_{y} a_{\perp}\right\}, z\right\} \rightarrow\left\{\mathbf{j}=\left\{j_{x}, j_{y}\right\}, z / \xi_{\|}\right\}$. The last term in Eq.(1) describes the Josephson-coupling with the coupling energy $E_{\mathbf{j}, \mathbf{j}+\mathbf{g}}$ between the wires, which is minimal for a coherent tunneling $\left(\varphi_{\mathbf{j}}(z) \sim \varphi_{\mathbf{j}+\mathbf{g}}(z)\right)$ of Cooper pairs between neighboring wires.

Fluctuations of the order parameter modulus can be neglected for pure superconductors ${ }^{34}$ far from $T_{c}^{(1)}$, satisfying the condition $\left(T_{c}^{(1)}-T\right) / T_{c}^{(1)} \gg n^{-2 / 3}$, where $T_{c}^{(1)}$ is the mean-field critical temperature calculated for an isolated wire and $n$ is the number of bands in each chain $^{40}$. Therefore, the contributions to the free energy functional (1), coming from the modulus of the order parameter $\left|\Delta_{\mathbf{j}}\right|$, are omitted.

We assume the Josephson energy $E_{\mathbf{j}, \mathbf{j}+\mathbf{g}}$ to be a random parameter with Gaussian distribution, centered at the mean value $E_{\mathbf{g}}$, given by

$$
P\left\{E_{\mathbf{j}, \mathbf{j}+\mathbf{g}}\right\}=\frac{1}{\sqrt{2 \pi W^{2}}} \exp \left\{-\frac{\left(E_{\mathbf{j}, \mathbf{j}+\mathbf{g}}-E_{\mathbf{g}}\right)^{2}}{2 W^{2}}\right\} .
$$

The variance $W^{2}$ is taken as a measure of disorder strength in this coupling of nearest neighbor chains. Employing the replica trick one can calculate the average value of the free energy $\mathcal{F}=-T\langle\ln Z\rangle$ over disorder. As usual we use $\langle\ln Z\rangle=\lim _{n \rightarrow 0} \frac{\partial}{\partial n}\left\langle Z^{n}\right\rangle$ and, in addition, express the n-th power of the partition function $Z=\int \prod_{\mathbf{j}} \mathcal{D} \varphi_{\mathbf{j}} e^{-F_{s t} / T}$ by means of replicated fields $\varphi^{a}$, $a=1 \ldots n$, as $($ for $\mathbf{B}=0)$

$$
\begin{aligned}
& \left\langle Z^{n}\right\rangle=\int \prod \mathcal{D} \varphi_{\mathbf{j}}^{a} \exp \left\{-\frac{N_{s} \xi_{\|}}{T} \sum_{\mathbf{j}, a} \int d z\left[\frac{\hbar^{2}}{8 m_{\|} \xi_{\|}^{2}}\left(\frac{\partial \varphi_{\mathbf{j}}^{a}}{\partial z}\right)^{2}\right.\right. \\
& +\sum_{\mathbf{g}} E_{\mathbf{g}}\left[1-\cos \left(\varphi_{\mathbf{j}}^{a}-\varphi_{\mathbf{j}+\mathbf{g}}^{a}\right)\right]+\frac{1}{2} \sum_{\mathbf{j}, \mathbf{g}}\left[\frac{N_{s} \xi_{\|} W}{T} \times\right. \\
& \left.\left.\times \int d z \sum_{a}\left[1-\cos \left(\varphi_{\mathbf{j}}^{a}-\varphi_{\mathbf{j}+\mathbf{g}}^{a}\right)\right]\right]^{2}\right\}
\end{aligned}
$$

The quadratic cosine term is linearized with the help of a Hubbard-Stratonovich transformation by introducing an auxiliary field $\zeta_{\mathbf{j}, \mathbf{g}}$. As a result, the sum over the replica variable $a$ is factorized and the replica limit can be performed, yielding for the averaged free energy

$$
\begin{array}{r}
\mathcal{F}=-T \int \prod_{\mathbf{j}, \mathbf{g}} \frac{N_{s}^{(1)} \xi_{\|}}{\sqrt{2 \pi}} d \zeta_{\mathbf{j}, \mathbf{g}} e^{-\frac{N_{s}^{(1) 2} \xi_{\Pi}^{2}}{2} \zeta_{\mathbf{j}, \mathbf{g}}^{2} \times} \\
\times \ln \int \prod \mathcal{D} \varphi_{\mathbf{j}} e^{-F / T} \\
\text { with } \quad F=N_{s}^{(1)} \xi_{\|} \sum_{\mathbf{j}} \int d z\left\{\frac{\hbar^{2}}{8 m_{\|} \xi_{\|}^{2}}\left(\frac{\partial \varphi_{\mathbf{j}}}{\partial z}\right)^{2}+\right. \\
\left.\sum_{\mathbf{g}}\left(E_{\mathbf{g}}-N_{s}^{(1)} \xi_{\|} W \zeta_{\mathbf{j}, \mathbf{g}}\right)\left[1-\cos \left(\varphi_{\mathbf{j}}-\varphi_{\mathbf{j}+\mathbf{g}}\right)\right]\right\} .
\end{array}
$$

The average value of a given functional $C\left(\left\{\varphi_{\mathbf{j}}\right\}\right)$, e.g. $\cos \varphi_{\mathbf{j}}$ or $\cos \left(\varphi_{\mathbf{j}}-\varphi_{\mathbf{j}+\mathbf{g}}\right)$, can be obtained according to the relation $\left\langle\left\langle C\left(\left\{\varphi_{\mathbf{j}}\right\}\right)\right\rangle\right\rangle=-\left.T \frac{\delta}{\delta \eta_{\mathbf{j}}}\langle\ln Z\rangle\right|_{\eta_{\mathbf{j}}=0}$ by adding the source term $\sum_{\mathbf{j}} \int d z \eta_{\mathbf{j}} C\left(\left\{\varphi_{\mathbf{j}}\right\}\right)$ to the free energy functional, which yields for the correlator

$$
\begin{aligned}
& \left\langle\left\langle C\left(\left\{\varphi_{\mathbf{j}}\right\}\right)\right\rangle\right\rangle=\int \prod_{\mathbf{j}, \mathbf{g}} \frac{N_{s}^{(1)} \xi_{\|}}{\sqrt{2 \pi}} d \zeta_{\mathbf{j}, \mathbf{g}} e^{-\frac{N_{s}^{(1) 2} \xi_{\|}^{2}}{2} \zeta_{\mathbf{j}, \mathbf{g}}^{2} \times} \\
& \times \frac{\int \mathcal{D} \varphi C\left(\left\{\varphi_{\mathbf{j}}\right\}\right) e^{-F / T}}{\int \mathcal{D} \varphi e^{-F / T}}
\end{aligned}
$$

where the double bracket $\langle\langle\ldots\rangle\rangle$ means averaging over thermodynamic fluctuations and over randomness. In order to estimate an asymptotic behavior of the correlator, e.g. $\left\langle\left\langle\cos \left(\varphi_{\mathbf{j}}-\varphi_{\mathbf{j}+\mathbf{g}}\right)\right\rangle\right\rangle$ we write the integrand of Eq.(6) as $\exp \left\{-N_{s}^{(1) 2} \xi_{\|}^{2} f\left(\zeta_{\mathbf{j}, \mathbf{g}}\right)\right\}$, and apply the stationary-phase approximation to determine an extremal value of the auxiliary field $\bar{\zeta}_{\mathbf{j}, \mathbf{g}}$ minimizing the function $f\left(\zeta_{\mathbf{j}, \mathbf{g}}\right)$. The minimal value of $\zeta_{\mathbf{j}, \mathbf{g}}$ is obtained to be

$$
\begin{array}{r}
\bar{\zeta}_{\mathbf{j}, \mathbf{g}}=\frac{W}{T} \int d z\left\{\left\langle\cos \left(\varphi_{\mathbf{j}}(z)-\varphi_{\mathbf{j}+\mathbf{g}}(z)\right)\right\rangle-\right. \\
\left.-\frac{\left\langle\cos \left(\varphi_{\mathbf{j}}(z)-\varphi_{\mathbf{j}+\mathbf{g}}(z)\right) \cos \left(\varphi_{\mathbf{j}}(0)-\varphi_{\mathbf{j}+\mathbf{g}}(0)\right)\right\rangle}{\left\langle\cos \left(\varphi_{\mathbf{j}}(0)-\varphi_{\mathbf{j}+\mathbf{g}}(0)\right)\right\rangle}\right\} .
\end{array}
$$

The constant $N_{s}^{(1)} \xi_{\|}$on the exponent can be estimated to be equal to $N_{s}^{(1)} \xi_{\|} \simeq \frac{\epsilon_{F}}{T_{c}^{(1)}} \sim 10^{3}$ for the organic superconductors with $\epsilon_{F}$ being the Fermi energy, which ensures a sharply peaked saddle point of the integrand. The thermodynamic averages in the expression of $\bar{\zeta}_{\mathbf{j}, \mathbf{g}}$ are taken with the free energy functional, given by Eq.(5), at the saddle point $\zeta_{\mathbf{j}, \mathbf{g}}=\bar{\zeta}_{\mathbf{j}, \mathbf{g}}$. So, a contribution of the nonmagnetic randomness to the effective free-energy functional is proportional to the variance of the phase cor(3)relator, which gives an idea on the form of the disorderdependent term in the effective functional. Note also that the saddle point for the averaged free energy $\mathcal{F}$ is given as $\tilde{\zeta}_{\mathbf{j}, \mathbf{g}}=-\frac{W}{\mathcal{F}} \int d z\left\langle\left[1-\cos \left(\varphi_{\mathbf{j}}(z)-\varphi_{\mathbf{j}+\mathbf{g}}(z)\right)\right]\right\rangle$, where $\tilde{\mathcal{F}}$ is the value of the free energy at the saddle point.

The critical temperature for the quasi-1D superconductors can now be found from Eq.(6), written for $\cos \varphi_{\mathbf{j}}$ by using the self-consistent mean-field method ${ }^{34}$, which 
consists in replacing the phase correlations of the cosine term by

$$
\begin{array}{ll}
\sum_{\mathbf{g}} & E_{\mathbf{g}}\left[1-\cos \left(\varphi_{\mathbf{j}}(z)-\varphi_{\mathbf{j}+\mathbf{g}}(z)\right)\right] \\
\longrightarrow & E_{\perp}[1-\langle\langle\cos (\varphi)\rangle\rangle \cos (\varphi(z))]
\end{array}
$$

where $E_{\perp}=\sum_{\mathbf{g}} E_{\mathbf{g}}$. For a clean system $\langle\langle\cos (\varphi)\rangle\rangle_{\text {eff }}$ was chosen ${ }^{34}$ to be equal to $\langle\cos (\varphi)\rangle$. For the disordered superconductor we choose $\langle\langle\cos \varphi\rangle\rangle_{\text {eff }}=\langle\langle\cos \varphi\rangle\rangle-$ $N_{s}^{(1)} \xi_{\|} \frac{\left\langle\langle\cos \varphi\rangle^{2}\right\rangle-\langle\langle\cos \varphi\rangle\rangle^{2}}{\langle\langle\cos \varphi\rangle\rangle}$. The functional integral over the phases in Eq.(6) can not yet be evaluated, even after this simplification. Taking advantage of the smallness of $\left(E_{\perp}-N_{s}^{(1)} \xi_{\|} W \zeta\right)\langle\langle\cos (\varphi)\rangle\rangle_{\text {eff }}$ near $T_{c}$, we expand both the numerator and the denominator of the integrand of Eq.(6), written for $\langle\langle\cos (\varphi)\rangle\rangle_{e f f}$, in this parameter. The thermodynamic averages become pure one-dimensional after this expansion, which can be taken easily, yielding a power series of $\zeta$ for the integrand. Therefore, the integration over $\zeta$ is immediately performed. Since all higher order in $\langle\langle\cos (\varphi)\rangle\rangle_{\text {eff }}$ terms of the expansion vanish at $T=T_{c}$, we get the equation for $T_{c}$

$$
\begin{array}{r}
1=\frac{E_{\perp} N_{s}^{(1)} \xi_{\|}}{T_{c}}\left(1-\frac{W^{2} \xi_{\|} N_{s}^{(1)} \eta^{2}}{T_{c} E_{\perp}}\right) \times \\
\quad \times \int\langle\cos (\varphi(0)) \cos (\varphi(z))\rangle d z,
\end{array}
$$

where $\eta$ is the coordination number. The phase-phase correlator in Eq.(9) is calculated in the clean limit of the $1 D$ free energy functional, obtained from Eq.(1) by setting $E_{\mathbf{j}, \mathbf{j}+\mathbf{g}}=0$, which returns (see for example Ref. ${ }^{39}$ )

$$
\langle\cos (\varphi(0)) \cos (\varphi(z))\rangle=\exp \left\{-|z| / r_{c}\right\}
$$

where $r_{c}=\hbar^{2} N_{s}^{(1)}(T) / 2 m_{\|} \xi_{\|} T$. Introducing a dimensionless $T_{c}$-shift by

$$
t=\sqrt{\eta \epsilon_{F} E_{\perp}}\left(\frac{1}{T_{c}}-\frac{1}{T_{c}^{(1)}}\right),
$$

with $\epsilon_{F}$ being the Fermi energy, and a dimensionless disorder parameter

$$
q=\frac{W^{2}}{E_{\perp}} \sqrt{\frac{2 m_{\|} \xi_{\|}^{2} \eta}{\hbar^{2} E_{\perp}}}=\frac{W^{2}}{2 E_{\perp} T_{c}^{(1)}} \sqrt{\frac{\eta \epsilon_{F}}{E_{\perp}}}
$$

Eqs.(9), (10) yield

$$
1=t^{2}(1-q t)
$$

The full solution of Eq.(13) has three roots, among which the physical one is confined to the finite $q$-range as shown by the bold line in Fig.1. A (physical) solution exists thus only within the finite range between clean limit $(C L)$ and dirty limit $(D L)$. One may expand and control this physical solution (of the cubic equation) in

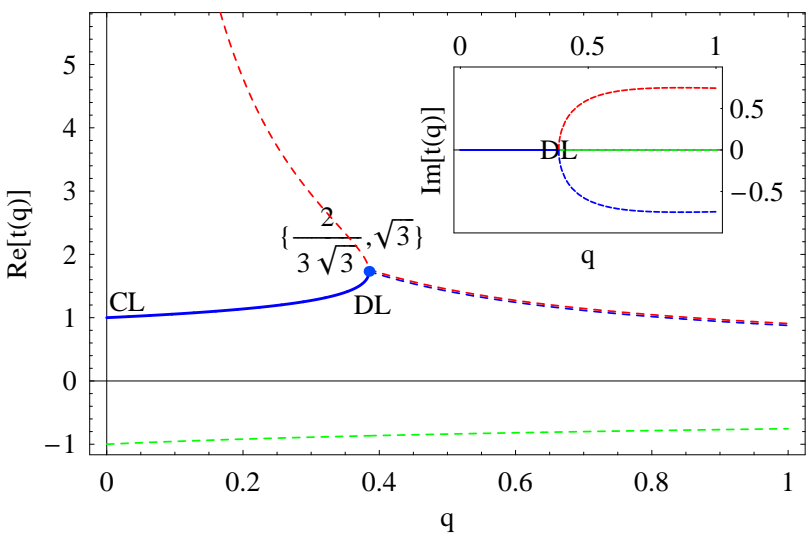

FIG. 1: The physical solution $t(q)$, giving the $T_{c}(W)$ dependence, within the full range from clean limit (CL: $q=0$ ) to the dirty limit (DL: $\left.q_{c}=2 / 3 \sqrt{3}\right)$ ) is highlighted as the bold (blue) curve. Formal solutions of the cubic Eq.(13) are shown for completeness. $T_{c}(q)$ vanishes abruptly at $q=q_{c}$.

the weak disorder regime (small $q$ ), where the $T_{c}$-shift obeys

$$
\frac{1}{T_{c}}=\frac{1}{T_{c}^{(1)}}+\frac{1}{\sqrt{\eta \epsilon_{F} E_{\perp}}}+\frac{1}{T_{c}^{(1)}}\left(\frac{W}{2 E_{\perp}}\right)^{2},
$$

showing that $T_{c}$ decreases with increasing randomness like $W^{2}$. For a pure system Eq.(14) gives the dependence $T_{c} \sim E_{\perp}^{1 / 2}$, in agreement with Efetov and Larkin in Ref. 34. This expression shows that even a small interchaincoupling sustains ODLRO in the system and, consequently, the critical temperature increases with $\sqrt{E_{\perp}}$. On the other hand, the competing destructive effect of disorder reduces $T_{c}$ due to "melting" of the order parameter phase coherence between neighboring chains.

According to the (physical) solution of Eq.(13), the critical temperature decreases monotonically with increasing $q$ but finite $T_{c}$ are confined to the interval $0 \leq q \leq q_{c}=\frac{2}{3 \sqrt{3}}$. The SC-phase becomes fully suppressed for disorder-strengths $W^{2}$ exceeding a critical disorder-value $W_{c}^{2}$ given by

$$
W_{c}^{2}=\frac{4 E_{\perp} T_{c}^{(1)}}{3} \sqrt{\frac{E_{\perp}}{3 \eta \epsilon_{F}}},
$$

beyond which the system is in a normal metallic phase (for $W^{2}>W_{c}^{2}$ ). The critical temperature drops to zero at $W^{2}=W_{c}^{2}$ with a jump of size

$$
\Delta T_{c}=T_{c}^{*}=\left(\sqrt{\frac{3}{\eta \epsilon_{F} E_{\perp}}}+\frac{1}{T_{c}^{(1)}}\right)^{-1} .
$$

Thus the SC-normal metal phase transition appears as a 1st-order transition.

In order to describe the behavior of $t$ near the disorder limit $D L$ (see Fig.1) we expand around $\left\{t^{*}, q^{*}\right\}=$ $\left\{\sqrt{3}, \frac{2}{3 \sqrt{3}}\right\}$, in terms of small (nonnegative) $\delta t=t^{*}-t$ and $\delta q=q^{*}-q$, which gives $\delta t=3^{3 / 4} \sqrt{\delta q}$. 
Near the dirty limit, the $T_{c}$-variation has an infinite slope (see also Fig.1). This can be reexpressed in terms of the physical parameters $\delta T_{c}=T_{c}-T_{c}^{*}$ and the variance $W^{2}$ of the Josephson-coupling, by reinserting Eqs.(12,14), as

$$
\delta T_{c}=\frac{3^{3 / 4} T_{c} T_{c}^{*}}{E_{\perp}\left(4 \eta \epsilon_{F} E_{\perp}\left(T_{c}^{(1)}\right)^{2}\right)^{1 / 4}}\left(W_{c}^{2}-W^{2}\right)^{1 / 2}
$$

in the vicinity of the breakdown point

$$
\left\{T_{c}^{*}, W_{c}^{2}\right\}=\left\{\left(\sqrt{\frac{3}{\eta \epsilon_{F} E_{\perp}}}+\frac{1}{T_{c}^{(1)}}\right)^{-1}, \frac{4 E_{\perp} T_{c}^{(1)}}{3} \sqrt{\frac{E_{\perp}}{3 \eta \epsilon_{F}}}\right\} .
$$

Thus the critical temperature decreases with disorder almost linearly but, approaching the dirty limit $D L$, it finally turns into a (nonanalytic) square-root behavior close to the breakdown point. In the absence of the non-magnetic disorder, even arbitrarily small Josephsoncoupling between the chains stabilizes the ODLRO and gives a non-zero critical temperature. However, the SC phase with finite Josephson-coupling can be destroyed by increasing the strength of non-magnetic disorder.

\section{QUANTUM PHASE FLUCTUATIONS}

The self-consistent mean-field method, applied above for the classical phase-fluctuation regime, expressed the $T_{c}$-equation in terms of the pure $1 \mathrm{D}$ phase-correlator (10), neglecting in this respect the Josephson-coupling between neighboring chains. We shall now improve the calculation of the phase-correlator by taking into account the transverse rigidity of the system, which provides a more realistic determination of the transition temperature in the quantum fluctuation regime. Our calculations are carried out in the Hamilton formalism for convenience, yet the problem can be formulated in the path integral language ${ }^{34}$ as well.

Let us start from the Lagrangian, again taking $\mathbf{B}=0$ for simplicity,

$$
\mathcal{L}=\frac{K \xi_{\|}(0)}{8} \sum_{\mathbf{j}} \int d z\left[\hbar \dot{\varphi}_{\mathbf{j}}(z)\right]^{2}-F_{\text {st }}^{e f f}\{\varphi\}
$$

where $\dot{\varphi}$ denotes the time derivative of the phase. The dynamical term in the Lagrangian can be interpreted as the electrostatic energy of charged wires ${ }^{35,38}$

$$
E_{e l}=\frac{1}{2} \sum_{\mathbf{i}, \mathbf{j}} \int d z \int d z^{\prime} C_{\mathbf{i}, \mathbf{j}}\left(z-z^{\prime}\right) V_{\mathbf{i}}(z) V_{\mathbf{j}}\left(z^{\prime}\right),
$$

generated according to the first Josephson equation $\dot{\varphi}=$ $(2 e / \hbar) V$, and $C_{\mathbf{i}, \mathbf{j}}\left(z-z^{\prime}\right)$ are the specific coefficients of electrostatic induction. Rewriting the electrostatic energy $E_{e l}$ in terms of the time-derivative of phases, the Fourier transform $K\left(\mathbf{q}_{\perp}, q_{z}\right)$ of the coefficients $K_{\mathbf{i}, \mathbf{j}}(z-$ $\left.z^{\prime}\right)=\frac{1}{4 e^{2}} C_{\mathbf{i}, \mathbf{j}}\left(z-z^{\prime}\right)$, has the physical meaning of a compressibility. In Eq.(18) we neglect dispersion in the compressibility and assume $K\left(\mathbf{q}_{\perp}, q_{z}\right)=K=$ const. This approximation is equivalent to a replacement of the electrostatic energy (19) by $\frac{1}{2} C \int d z \sum_{j} V_{j}^{2}(z)$. The parameter $K$ can be calculated ${ }^{34}$ in the presence of Coulomb screening for a small Born parameter $\frac{e^{2}}{\hbar v_{0}} n<1$, which results in

$$
K=\frac{n}{\pi \hbar v_{0}}\left[1+\frac{e^{2}}{\pi \hbar v_{0}} n \ln \frac{a_{\perp}}{d}\right]^{-1} .
$$

There, $K_{0}=\frac{n}{\pi \hbar v_{0}}$ is the unscreened compressibility, $v_{0}$ denotes the longitudinal velocity of an electron on the Fermi surface averaged over $n$ subbands, while $a_{\perp}$ and $d$ stand for the interchain-distance and the diameter of a superconducting wire, respectively.

In order to get $F_{s t}^{e f f}\{\varphi\}$ we average the free energy over disorder and apply a mean-field approximation, corresponding to the replacement $\int d z \int d z^{\prime}\left[1-\cos \left(\varphi_{\mathbf{j}}(z)-\right.\right.$ $\left.\left.\varphi_{\mathbf{j}+\mathbf{g}}(z)\right)\right]\left[1-\cos \left(\varphi_{\mathbf{j}}\left(z^{\prime}\right)-\varphi_{\mathbf{j}+\mathbf{g}}\left(z^{\prime}\right)\right)\right]$ by $\left\langle\left\langle\left[1-\cos \left(\varphi_{\mathbf{j}}(z)-\right.\right.\right.\right.$ $\left.\left.\left.\left.\varphi_{\mathbf{j}+\mathbf{g}}(z)\right)\right]\right\rangle\right\rangle \int d z\left[1-\cos \left(\varphi_{\mathbf{j}}(z)-\varphi_{\mathbf{j}+\mathbf{g}}(z)\right)\right]$.

The Hamiltonian, expressed through the phases $\varphi_{\mathbf{j}}$ and canonical conjugate momenta $\Pi_{\mathbf{j}}$, becomes

$$
\begin{aligned}
\mathcal{H}=\sum_{\mathbf{j}} \int d & \left\{2 \frac{\Pi_{\mathbf{j}}^{2}(z)}{K \xi_{\|}(0)}+\frac{\hbar^{2} N_{s}^{(1)}(T)}{8 m_{\|} \xi_{\|}}\left[\left(\frac{\partial \varphi_{\mathbf{j}}}{\partial z}\right)^{2}+\right.\right. \\
+ & \left.\left.\sum_{\mathbf{g}} \delta_{c l}^{2}\left[1-\cos \left(\varphi_{\mathbf{j}}(z)-\varphi_{\mathbf{j}+\mathbf{g}}(z)\right)\right]\right]\right\},
\end{aligned}
$$

where $\Pi_{\mathbf{j}}=\frac{1}{\hbar} \frac{\delta \mathcal{L}}{\delta \dot{\varphi}_{\mathbf{j}}}=\frac{1}{4} \hbar K \xi_{\|}(0) \dot{\varphi}_{\mathbf{j}}$, while $\delta_{c l}$ is given by

$$
\delta_{c l}^{2}=\delta_{0}^{2}\left[1-\frac{W^{2} N_{s}^{(1)} \xi_{\|}}{E_{\perp} T}\left\langle\left\langle\left[1-\cos \left(\varphi_{\mathbf{j}}(z)-\varphi_{\mathbf{j}+\mathbf{g}}(z)\right)\right]\right\rangle\right\rangle\right]
$$

and represents the dimensionless anisotropy-parameter or the transverse rigidity of the system; $\delta_{0}$ in Eq.(22) is transverse rigidity of the pure system

$$
\delta_{0}=\left(\frac{E_{\perp}}{\hbar^{2} / 8 m_{\|} \xi_{\|}^{2}}\right)^{1 / 2}=\frac{\left(\epsilon_{F} E_{\perp}\right)^{1 / 2}}{T_{c}^{(1)}}
$$

The phase dynamics in the classical limit can be obtained from the Euler-Lagrange equation, which is described by a set of coupled sine-Gordon type non-linear equations

$$
\ddot{\varphi}_{\mathbf{j}}(z)=\bar{\omega}^{2}\left\{\frac{\partial^{2} \varphi_{\mathbf{j}}}{\partial z^{2}}-\sum_{\mathbf{g}} \delta_{c l}^{2} \sin \left(\varphi_{\mathbf{j}}(z)-\varphi_{\mathbf{j}+\mathbf{g}}(z)\right)\right\}
$$

where $\bar{\omega}$ is a characteristic scale of frequency, and

$$
\bar{\omega}^{2}=\frac{N_{s}^{(1 D)}(T)}{m_{\|} K \xi_{\|}^{2}}
$$


We express $\bar{\omega}$ as $\bar{\omega}=2 \pi \alpha \tau^{1 / 2} T_{c}^{(1)} / \hbar$, where

$$
\alpha=\frac{1}{2 \pi}\left(\frac{16}{K\left(\hbar^{2} N_{s}^{(1 D)}\right) / m_{\|}}\right)^{1 / 2} .
$$

The parameter $\alpha$ is the essential parameter of the theory, which can be written, using Eq.(20), as

$$
\alpha=\frac{1}{\sqrt{\pi n}}\left[1+\frac{e^{2}}{\pi \hbar v_{0}} n \ln \left(\frac{a_{\perp}}{d}\right)\right]^{1 / 2} .
$$

The system of equations (24) is linearized for small $\varphi_{\mathbf{j}}$ and its Fourier-transformation becomes diagonal

$$
\ddot{\varphi}\left(\mathbf{q}_{\perp}, q_{z}\right)=-\omega^{2}\left(\mathbf{q}_{\perp}, q_{z}\right) \varphi\left(\mathbf{q}_{\perp}, q_{z}\right)
$$

The eigenfrequency of oscillations $\omega\left(\mathbf{q}_{\perp}, q_{z}\right)$ is given in the harmonic approximation as

$$
\omega\left(\mathbf{q}, q_{z}\right)=\bar{\omega}\left[q_{z}^{2}+\delta_{c l}^{2} 2\left(2-\cos q_{x}-\cos q_{y}\right)\right]^{1 / 2} .
$$

These equations describe the frequency of low-lying plasmon-mode of the system.

The quantum description is realized by expressing $\varphi_{\mathbf{q}}$ and $\Pi_{\mathbf{q}}$ as a linear superposition of Bose operators $b_{\mathbf{q}}$ and $b_{\mathbf{q}}^{\dagger},\left[b_{\mathbf{q}}, b_{\mathbf{q}}^{\dagger}\right]=1$, by

$$
\begin{aligned}
\varphi_{\mathbf{q}} & =\left(\frac{\pi \alpha \bar{\omega}}{\omega(\mathbf{q})}\right)^{1 / 2}\left(b_{\mathbf{q}}+b_{-\mathbf{q}}^{\dagger}\right) \\
\Pi_{\mathbf{q}} & =i\left(\frac{\omega(\mathbf{q})}{4 \pi \alpha \bar{\omega}}\right)^{1 / 2}\left(b_{-\mathbf{q}}-b_{\mathbf{q}}^{\dagger}\right) .
\end{aligned}
$$

If we expand the cosine term in Eq.(21) up to the quadratic term and express the phases $\varphi_{\mathbf{q}}$ and the conjugate momentum $\Pi_{\mathbf{q}}$ in terms of creation and annihilation operators, we get the Hamiltonian in the harmonic approximation as

$$
\hat{\mathcal{H}}_{0}=\sum_{\mathbf{q}} \hbar \omega\left(\mathbf{q}_{\perp}, q_{z}\right)\left[b_{\mathbf{q}}^{\dagger} b_{\mathbf{q}}+\frac{1}{2}\right],
$$

where the energy spectrum is defined by Eq.(29).

In order to take into account the quantum effects in the Hamiltonian, we have to express the cosine term in Eq.(21) in a normal ordering before expanding over $b_{\mathbf{q}}^{\dagger}$ and $b_{\mathbf{q}}$

$$
\begin{gathered}
1-\cos \left(\varphi_{\mathbf{j}}(z)-\varphi_{\mathbf{j}+\mathbf{g}}(z)\right)= \\
1-\frac{1}{2} e^{-S_{\alpha}(\mathbf{g}, 0)}\left(e^{\sum_{\mathbf{q}} A_{\mathbf{q}} b_{\mathbf{q}}^{\dagger}} e^{-\sum_{\mathbf{q}} A_{\mathbf{q}}^{\star} b_{\mathbf{q}}}+\text { h.c. }\right),
\end{gathered}
$$

where

$$
A_{\mathbf{q}}(\mathbf{j}, \mathbf{g})=i\left(\frac{\pi \alpha \bar{\omega}}{N \omega(\mathbf{q})}\right)^{1 / 2} e^{i q_{z} z+i \mathbf{q}_{\perp} \mathbf{j}}\left(1-e^{i \mathbf{q} \perp \mathbf{g}}\right) .
$$

The prefactor $\exp \left\{-S_{\alpha}(\mathrm{g}, 0)\right\}$ originates in the commutation relation between $b_{\mathbf{q}}^{\dagger}$ and $b_{\mathbf{q}}$, and by taking into account the Baker-Campbell-Hausdorff relation $\exp \left(\hat{H}_{1}+\right.$ $\left.\hat{H}_{2}\right)=\exp \left(\hat{H}_{1}\right) \exp \left(\hat{H}_{2}\right) \exp \left\{-\frac{1}{2}\left[\hat{H}_{1}, \hat{H}_{2}\right]\right\}$. Furthermore

$$
S_{\alpha}(\mathbf{g}, 0)=\frac{1}{2} \sum_{\mathbf{q}}\left|A_{\mathbf{q}}(\mathbf{j}, \mathbf{g})\right|^{2}=\frac{\pi \alpha \bar{\omega}}{N} \sum_{\mathbf{q}} \frac{1-\cos \left(\mathbf{q}_{\perp} \mathbf{g}\right)}{\omega\left(\mathbf{q}_{\perp}, q_{z}\right)}
$$

where $N$ is the number of unit cells per volume.

It is clear from Eq.(32) that the physical meaning of $\exp \left\{-S_{\alpha}(\mathbf{g}, 0)\right\}$ is an average of $\cos \left(\varphi_{\mathbf{j}}(z)-\varphi_{\mathbf{j}+\mathbf{g}}(z)\right)$ over the ground state at $T=0$. In the framework of the selfconsistent phonon approximation (SCPA), we expand the Josephson term (32) in powers of the creation and annihilation operators, $b_{\mathbf{q}}^{\dagger}$ and $b_{\mathbf{q}}$ repsectively. Expressing the leading (harmonic) part of the Hamiltonian (21) in terms of the particle number operator $\hat{N}_{\mathbf{q}}=b_{\mathbf{q}}^{\dagger} b_{\mathbf{q}}$, we obtain again a harmonic Hamiltonian as in Eq. (31), $\hat{\mathcal{H}}_{0} \rightarrow \hat{\mathcal{H}}_{\alpha}^{(0)}(0)$, but with the different oscillation frequency

$$
\omega\left(\mathbf{q}, q_{z}\right)=\bar{\omega}\left[q_{z}^{2}+\delta_{c l}^{2} e^{-S_{\alpha}^{(0)}(\mathbf{g}, 0)} 2\left(2-\cos q_{x}-\cos q_{y}\right)\right]^{1 / 2},
$$

Thus the application of the SCPA results in a renormalization of the parameter of anisotropy $\delta_{c l}$ in the frequency of oscillation $\omega(\mathbf{q}, 0)$ by means of the phasephase correlator as

$$
\delta_{c l}^{2} \rightarrow \delta_{q u}^{2}(0)=\delta_{c l}^{2} e^{-S_{\alpha}(\mathbf{g}, 0)} .
$$

We note that in order to preserve a maximal coherence of phases at $\varphi_{\mathbf{j}}=\varphi_{\mathbf{j}+\mathbf{g}}$ in the Josephson term $1-\cos \left(\hat{\varphi}_{\mathbf{j}}(z)-\hat{\varphi}_{\mathbf{j}+\mathbf{g}}(z)\right)$ the latter is replaced in the framework of the SCPA by $e^{-S_{\alpha}(\mathbf{g}, 0)}-\cos \left(\hat{\varphi}_{\mathbf{j}}(z)-\hat{\varphi}_{\mathbf{j}+\mathbf{g}}(z)\right)$, which corresponds to shifting of the energy origin. Indeed, the Josephson term in the initial expression of the Hamilton function was introduced in a such way that it becomes zero for a maximal coherence of phases $\varphi_{\mathbf{j}}=\varphi_{\mathbf{j}+\mathbf{g}}$. Zero-point fluctuations at $T=0$ in the quantum case destroy the phase coherence and increase the Josephson energy. By shifting $1 \rightarrow e^{-S_{\alpha}(\mathbf{g}, 0)}$ we again reach a minimal Josephson energy at $T=0$ in the quantum case too. Similar shifting was done also in Eq.(22).

In the expansion of the exponential operator of Eq.(32) we select all diagonal terms, which can be expressed in terms of the bosonic particle number operator $\hat{N}_{\mathbf{q}}=$ $b_{\mathbf{q}}^{\dagger} b_{\mathbf{q}}{ }^{38}$. This yields

$$
\left\{\cos \left(\varphi_{\mathbf{j}}(z)-\varphi_{\mathbf{j}+\mathbf{g}}(z)\right)\right\}_{\text {diag }}=e^{-S_{\alpha}(\mathbf{g}, 0)} \prod_{\mathbf{q}}\{1-
$$

$\left.-\left|A_{\mathbf{q}}\right|^{2} b_{\mathbf{q}}^{\dagger} b_{\mathbf{q}}+\left|A_{\mathbf{q}}\right|^{4} \frac{\left(b_{\mathbf{q}}^{\dagger}\right)^{2}}{2 !} \frac{b_{\mathbf{q}}^{2}}{2 !}-\left|A_{\mathbf{q}}\right|^{6} \frac{\left(b_{\mathbf{q}}^{\dagger}\right)^{3}}{3 !} \frac{b_{\mathbf{q}}^{3}}{3 !} \pm \ldots\right\}$.

Absorbing now the product in Eq.(37) into the exponential form, and neglecting all higher orders in $\left|A_{\mathbf{q}}\right|^{2}$ beyond the leading term $\left|A_{\mathbf{q}}\right|^{2}$ (for justification see below [41]), 
we get

$$
\begin{array}{r}
\left\{\cos \left(\hat{\varphi}_{\mathbf{j}}-\hat{\varphi}_{\mathbf{j}+\mathbf{g}}\right)\right\}_{\text {diag }}=\exp \left\{-S_{\alpha}(\mathbf{g}, 0)-\sum_{\mathbf{q}}\left|A_{\mathbf{q}}\right|^{2} \hat{N}_{\mathbf{q}}\right\}= \\
e^{-S_{\alpha}(\mathbf{g}, T)} \equiv \exp \left\{-\frac{2 \pi \alpha \bar{\omega}}{N} \sum_{\mathbf{q}} \frac{1-\cos (\mathbf{q} \perp \mathbf{g})}{\omega(\mathbf{q})}\left(\hat{N}_{\mathbf{q}}+\frac{1}{2}\right)\right\} .
\end{array}
$$

Thus, after this step of calculations we still restrict ourself with harmonic approximation, describing the system by the Hamiltonian $\hat{\mathcal{H}}_{\alpha}^{(0)}(T)$ like $(31)$ where the transverse rigidity $\delta_{c l}^{2}$ in the frequency $\omega(\mathbf{q}, T)$ is renormalized as

$$
\delta_{c l}^{2} \rightarrow \delta_{q u}^{2}(T)=\delta_{c l}^{2} \exp \left\{-S_{\alpha}(\mathbf{g}, T)\right\}
$$

where

$$
\begin{aligned}
& \left\langle\left\langle\cos \left(\varphi_{\mathbf{j}}(z)-\varphi_{\mathbf{j}+\mathbf{g}}(z)\right)\right\rangle\right\rangle \equiv e^{-S_{\alpha}(\mathbf{g}, T)} \\
= & \frac{\operatorname{Tr}\left\{e^{-\beta \hat{H}_{\alpha}^{(0)}} \cos \left(\hat{\varphi}_{\mathbf{j}}(z)-\hat{\varphi}_{\mathbf{j}+\mathbf{g}}(z)\right)\right\}}{\operatorname{Tr}\left\{e^{-\beta \hat{\mathcal{H}}_{\alpha}^{(0)}}\right\}}
\end{aligned}
$$

The trace over the diagonal part of the phase-phase correlator within the harmonic approximation replaces the bosonic filling number operator $\hat{N}_{\mathbf{q}}$ by the Planck distribution function for phonons with energies of $\hbar \omega(\mathbf{q}, T)$ as $\hat{N}_{\mathbf{q}} \rightarrow\left\{\exp \left(\frac{\hbar \omega(\mathbf{q}, T)}{T}\right)-1\right\}^{-1}$, yielding the following expression for the $S_{\alpha}(\mathbf{g}, T)$

$$
S_{\alpha}(\mathbf{g}, T)=\frac{\pi \alpha \bar{\omega}}{N} \sum_{\mathbf{q}} \frac{1-\cos \left(\mathbf{q}_{\perp} \mathbf{g}\right)}{\omega(\mathbf{q}, T)} \operatorname{coth}\left(\frac{\hbar \omega(\mathbf{q}, T)}{T}\right) .
$$

The correlator $e^{-S_{\alpha}(\mathbf{g}, T)}$ and its $T=0$-limit, as given by Eqs.(40) and (34) respectively, are evaluated explicitly in the Appendix.

\section{A. Quantum Criticality at $\mathbf{T}=\mathbf{0}$}

The zero-temperature behavior of the system is analyzed by means of the phase-phase correlator $e^{-S_{\alpha}(\mathbf{g}, 0)}$, the explicit expression for which is given by Eq.(59) in the Appendix. Expressing the phase-phase correlator $e^{-S_{\alpha}(\mathbf{g}, 0)}$ in terms of $S_{\alpha}(\mathbf{g}, 0)$ gives $e^{-S_{\alpha}(\mathbf{g}, 0)}=$ $\left(\delta_{q u}(0)\right)^{\alpha} \equiv \delta_{q u}^{\alpha}$, which implies that even a small interchain-coupling stabilizes ODLRO in the system, hence also a finite $T$ phase transition should exist. In order to get an explicit expression for the dependence of $\delta_{q u}$ on $\delta_{0}$ and on disorder, we have to solve the equation $\delta_{q u}^{2}=\delta_{c l}^{2} e^{-S_{\alpha}(\mathbf{g}, 0)}$ together with Eq.(22) for $\delta_{c l}$. Thus the equation for the reduced transverse rigidity $\delta_{q u}^{*}=\delta_{q u} / \delta_{q u}^{(0)}$, where $\delta_{q u}^{(0)}=\delta_{0}^{\frac{2}{2-\alpha}}$ is the renormalized transverse rigidity for the clean system at $T=0$, assumes the form

$$
\left(\delta_{q u}^{*}\right)^{3-2 \alpha}=\left(\delta_{q u}^{*}\right)^{1-\alpha}-q_{q u},
$$

where the quantum parameter of randomness $q_{q u}$ reads

$$
q_{q u}=\frac{C W^{2}}{2 E_{\perp}^{2}} \delta_{0}^{\frac{2}{2-\alpha}} .
$$

$C$ in Eq. (42) is a constant $C \sim 1$. Although Eqs.(13) and (41) are written for two different characteristic parameters of the system, it is easy to see that the equation for $\delta_{q u}^{*}(0)$, if we neglect the quantum effects at $\alpha=0$, coincides with Eq.(13) written for $y=1 / t$.

Eq.(41) can be solved approximately for moderately weak disorder, yielding the following expression for $\delta_{q u}(0)$

$$
\delta_{q u}(0)=\delta_{0}^{\frac{1}{1-\alpha / 2}}\left[1-C \frac{1-\alpha}{2-\alpha}\left(\frac{W}{E_{\perp}}\right)^{2} \delta_{0}^{\frac{1}{1-\alpha / 2}}\right]^{\frac{1}{1-\alpha}} .
$$

Hence, the evaluation of the phase-phase correlator $\left.\left\langle\left\langle\cos \left(\varphi_{\mathbf{j}}(z)-\varphi_{\mathbf{j}+\mathbf{g}}(z)\right)\right\rangle\right\rangle\right|_{T=0}=e^{-S_{\alpha}(\mathbf{g}, 0)}$ in the presence of disorder yields the result

$$
\begin{aligned}
& \left.\left\langle\left\langle\cos \left(\varphi_{\mathbf{j}}(z)-\varphi_{\mathbf{j}+\mathbf{g}}(z)\right)\right\rangle\right\rangle\right|_{T=0}=\left(\frac{\epsilon_{F} E_{\perp}}{T_{c}^{(1)^{2}}}\right)^{\frac{\alpha}{2-\alpha}} \times \\
& \times\left[1-C \frac{1-\alpha}{2-\alpha}\left(\frac{W}{E_{\perp}}\right)^{2}\left(\frac{\epsilon_{F} E_{\perp}}{T_{c}^{(1)^{2}}}\right)^{\frac{1}{2-\alpha}}\right]^{\frac{\alpha}{1-\alpha}} \cdot
\end{aligned}
$$

In the absence of the disorder, i.e. for $W=0$, we retrieve the phase-phase correlator

$$
\left.\left\langle\cos \left(\varphi_{\mathbf{j}}(z)-\varphi_{\mathbf{j}+\mathbf{g}}(z)\right)\right\rangle\right|_{T=0, W=0}=\left(\frac{\epsilon_{F} E_{\perp}}{T_{c}^{(1)^{2}}}\right)^{\frac{\alpha}{2-\alpha}},
$$

as obtained by Efetov and Larkin ${ }^{34}$ for pure quasi-1D superconductors. The critical temperature for a quasi-1D superconductor, according to Efetov and Larkin ${ }^{34}$, is defined by $T_{c 0}=\delta_{q u}^{(0)}(0) T_{c}^{(1)}$ for pure superconductors and by $T_{c 0}=\delta_{q u}(0) T_{c}^{(1)}$ in our case for dirty superconductors. This relation with Eq.(44) shows that $T_{c 0}$ decreases nonlinearly with disorder.

The numerical solution of Eq.(41) is depicted in Fig.2. The reduced $T=0$ transverse rigidity $\left.\delta_{q u}^{*}\left(q_{q u}\right)\right|_{T=0}$ is shown to decrease with increasing disorder for (fixed) $\alpha<1$, and suddenly drops to zero at the critical disorder strength $q_{q u}=q_{q u}^{c}$. Hence the quantum critical behavior corresponds to a first order phase transition. Fig.2 shows how the breakdown point shifts with increasing $\alpha$ to higher values of the randomness, and the jump vanishes as $\alpha \rightarrow 1$. Eq.(41) becomes linear for $\alpha=1$ and gives, by inferring the $q_{q u}(W)$-relation from Eq.(42),

$$
\left.\delta_{q u}(W)\right|_{T=0, \alpha=1}=\delta_{0}^{2}\left[1-\frac{C W^{2} \epsilon_{F}}{2 E_{\perp}\left(T_{c}^{(1)}\right)^{2}}\right] .
$$

Here, the transverse rigidity $\left.\delta_{q u}(W)\right|_{T=0, \alpha=1}$ decreases 


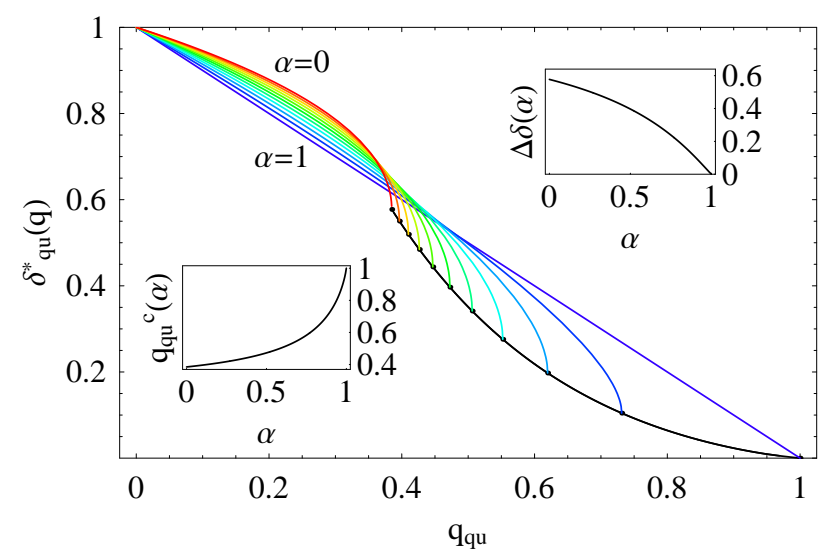

FIG. 2: The dependence of the reduced $T=0$ transverse rigidity $\delta_{q u}^{*}\left(q_{q u}\right)$ on the disorder-strength parameter $q_{q u}$ is shown for $0 \leq \alpha \leq 1$ in steps of $\Delta \alpha=0.1$. At $q_{q u}=q_{q u}^{c}$, $\delta_{q u}^{*}\left(q_{q u}\right)$ drops to zero for $\alpha<1$ and vanishes continuously only at $\alpha=1$. Inserts show the $\alpha$-variation of the jump (upper right corner) and of its position $q_{q u}^{c}(\alpha)$ (lower left).

linearly with increasing $W^{2}$ and vanishes at

$$
W_{c}^{2}=\frac{2 E_{\perp}\left(T_{c}^{(1)}\right)^{2}}{C \epsilon_{F}} .
$$

The quantum critical behavior in the model is however controlled by two parameters, the strength of randomness $q_{q u}(W)$ and the parameter of quantum dynamics $\alpha$. For $\alpha<1$, the superconductor-normal metal phase transition at $T=0$ is always discontinuous, and only turns into second-order at $\alpha=1$.

\section{B. Phase transition at finite temperatures}

Let us now study the finite $T$ behavior of the transverse rigidity. The phase transition in a quasi-1D superconductor occurs at some temperature $T=T_{c}$ when the transverse rigidity in the ensemble of phases $\left\{\varphi_{\mathbf{j}}(z)\right\}$ vanishes, which results in "melting" of the phase coherence. The energy spectrum $\omega\left(\mathbf{q}_{\perp}, q_{z}\right)$ of the collective excitations is reorganized and the transverse $\mathbf{q}_{\perp}$-dependent part of $\omega\left(\mathbf{q}_{\perp}, q_{z}\right)$ vanishes at $T=T_{c}$, i.e. symmetry breaking occurs in the bosonic excitation at $T=T_{c}$. Inserting the solution of Eq.(34) for $T<\alpha T_{c}^{(1)}$ into $\delta_{q u}^{2}(T)=\delta_{c l}^{2} e^{-S_{\alpha}(\mathbf{g}, T)}$ and using Eq.(38), we obtain

$$
\delta_{q u}^{2}(T)=\delta_{q u}^{2}(0)\left(\frac{T}{\alpha T_{c 0}}\right)^{\alpha} \exp \left\{-C \frac{T}{T_{c 0}} \frac{\delta_{q u}(0)}{\delta_{q u}(T)}\right\}
$$

where a new temperature scale is introduced by means of $T_{c 0}=\delta_{q u}(0) T_{c}^{(1)}$. In terms of $y=\left(\frac{\alpha T_{c 0}}{T}\right)^{\alpha / 2} \frac{\delta_{q u}(T)}{\delta_{q u}(0)}$ and $\theta=\left(\frac{T}{T_{c 0}}\right)^{1-\frac{\alpha}{2}} \frac{C}{2} \alpha^{\alpha / 2}$, Eq.(47) assumes the form $y=\exp \{-\theta / y\}$, which has a non-zero solution only for

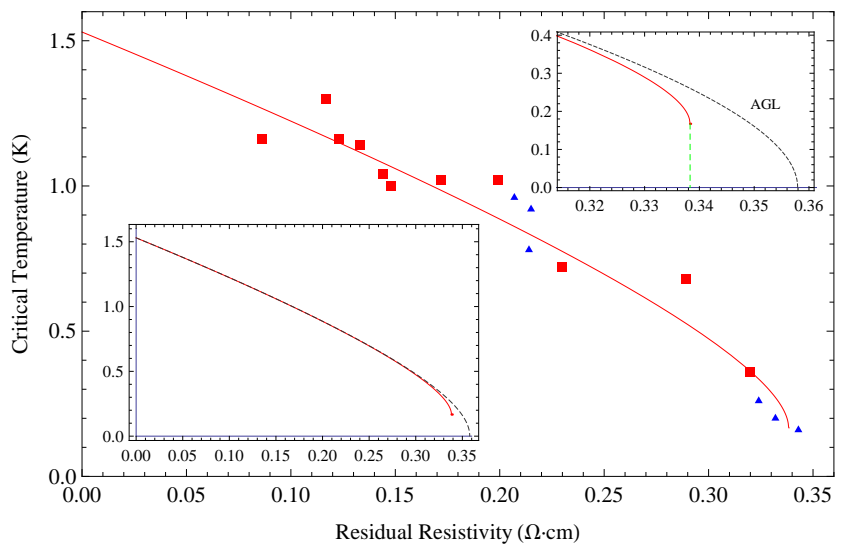

FIG. 3: Comparison of the $T_{c}$-decrease, as obtained from Eq.(48) with fit parameter $\alpha=0.47$ of the present theory (after proper rescaling), with experimental data imported from Ref.10 by Joo et al. Different symbols belong to different samples and different slow (squares) or fast (triangles) cooling procedures. In a first approximation the theoretical solid curve, as shown from the clean limit up to the dirty limit, fits and confirms the slightly but increasingly non-linear behavior with a final discontinuous drop of $T_{c}$ at the critical disorder. In the lower left inset $T_{c}$-curve employing the AbrikosovGor'kov-Larkin (AGL) digamma-function (dashed curve) for unconventional superconductors is shown for comparison with the present theory: the AGL-curve is chosen such that its value and slope at zero disorder agrees with the present one. The insert in the upper right corner shows the remarkable deviation of these two theoretical curves in the large disorder regime and close to the breakdown.

$\theta \leq e^{-1}$. The finite solution of this equation vanishes discontinuously at $\theta=\theta_{c}=e^{-1}$, giving the following value for $T_{c}$

$$
T_{c}=\delta_{q u}(0) T_{c}^{(1)} \alpha^{-\frac{\alpha}{2-\alpha}}(2 / e C)^{\frac{2}{2-\alpha}} .
$$

The magnitude of the jump in $y\left(\theta_{c}\right)$ is $e^{-1}$, and hence the phase transition is of first-order. The similar behavior has been found also in the planar rotor model ${ }^{42}$ in the absence of disorder. The dependence of $T_{c}$ on the disorder is determined by the zero-temperature transverse rigidity $\delta_{q u}(0)$, the behavior of which is depicted in Fig. 2. Therefore, for arbitrary $\alpha<1$ the critical temperature decreases monotonically with increasing randomness and drops to zero at the critical disorder strength $q_{q u}=q_{q u}^{c}$. The variation of the critical temperature versus the residual resistivity of the organic superconductor $\left(\mathrm{TMTSF}_{2}\left(\mathrm{ClO}_{4}\right)_{(1-x)}\left(\mathrm{ReO}_{4}\right)_{x}\right.$ has been explored by Joo et al. in Ref.9,10. The experimental data are read off from Ref.10 and copied into Fig. 3 in order to provide a close comparison with a theoretical fit-curve as obtained from Eq.(48) of the present theory.

In this first approach and within a moderate accuracy, the data as published in the paper of Joo et al., eventually appear to find an explanation by our theory. In order to establish the link between experiment and theory, the following argument is exploited: the substitution of $\mathrm{ClO}_{4}$ 
anions by $\mathrm{ReO}_{4}^{-}$in the relaxed (R) samples increases the residual resistivity, which is proportional to the inverse lifetime $1 / \tau$ of electron as well as to the disorder strength $W^{2}$ (as used in the present theory).

For the relaxed samples of Ref.10, referred to as the R-sample(s), the doping concentration $x$ varies in the interval of $0 \leq x \leq 0.1$ under slow cooling from the clean sample to the nominal concentration $x=0.1$. Slow cooling of $R$-samples assures a uniform orientation of the anions along the stacking axis, whereas fast cooling in the (quenched) $Q$-samples introduces strong orientational disorder and increases the residual resistivity. $T_{c}$ decreases quasi-linearly with increasing disorder (or the residual resistivity) in the large interval of the randomness. Around the breakdown point the dependence of $T_{c}$ on randomness is non-linear. The critical doping concentration, corresponding to the breakdown of the superconducting state, grows with the quantum charging effect in the system. Substitution of $\mathrm{ClO}_{4}$ anions by $\mathrm{ReO}_{4}$ seems to increase the quantum charging parameter $\alpha$, shifting thus the breakdown point to a higher value of the residual resistivity. All these features and experimental evidences agree well with the theory.

In order to compare with other well-known cases of $T_{c}$-suppression by disorder we consider the pair breaking theory for a superconductor with unconventional gap symmetry ${ }^{43,44}$. This physically different case of $T_{c^{-}}$ reduction by non-magnetic impurities in unconventional superconductors was found to be described by the famous digamma-formula of Abrikosov-Gor'kov's conventional pair breaking theory ${ }^{13}$ in the presence of paramagnetic impurities. The $T_{c}$-reduction may thus be expressed in the form

$$
\ln \left(\frac{e^{\Psi\left(\frac{1}{2}\right)} T_{c}^{(1)}}{T_{c}}\right)=\Psi\left(\frac{1}{2}+\frac{\rho T_{c}^{(1)}}{2 \pi T_{c}}\right),
$$

where $\Psi$ means the digamma function, $\rho=\hbar / 2 \tau T_{c}^{(1)}$ is the depairing parameter, and $\tau$ the elastic scattering time. Both theoretical curves, as shown in the insets of Fig.3, can be chosen to coincide for weak and moderately strong disorder (where the linear decay is rather unspecific). Approaching the SC-breakdown at larger disorder they differ however substantially. The AGL-solution for unconventional pairing approaches $T_{c}=0$ continuously and obeys a square root dependence $T_{c} \sim\left(\tilde{q}-\tilde{q}_{c}\right)^{1 / 2}$, where $\tilde{q}$ stands for the disorder-strength in the AGL-case. This square root law follows from the leading $\left(O\left(z^{2}\right)\right)$ correction of the digamma-function $\Psi\left(\frac{1}{2}+\frac{\tilde{q}}{z}\right)$ given by the Laurent series of its exponential

$$
\exp \left[\Psi\left(\frac{1}{2}+\frac{\tilde{q}}{z}\right)\right]=\frac{\tilde{q}}{z}+\frac{z}{24 \tilde{q}}+O\left(z^{2}\right)
$$

near the logarithmic branch point of $\Psi$ at infinity, hence $z=0$. In the physical context the variable $z$ corresponds to the critical temperature $T_{c}$ of Eq.(49). By comparing the exponential of Eq.(49) one can see that $z=T_{c}=0$ is reached for $\tilde{q}=\rho T_{c}^{(1)} /(2 \pi) \rightarrow \tilde{q}_{c}=\exp \left(\Psi\left(\frac{1}{2}\right)\right)$.
By contrast, the present theory does not allow for a continuous breakdown of superconductivity. According to Fig. 3 the suppression of $T_{c}$ is stronger and an abrupt breakdown occurs at $\left.T_{c}\right|_{\min }>0$. The numerical data show a square root behavior however near the minimal finite $T_{c}$.

The breakdown point in the curve, corresponding to our theory, seems to allow for the existence of an intermediate phase, perhaps a glassy phase below a tricritical point.

\section{MEISSNER EFFECT}

The current density is calculated according to $\frac{1}{c} \mathbf{J}(z, \mathbf{j})=-T \frac{\delta}{\delta \mathbf{A}}\langle\ln Z(\mathbf{A})\rangle$, where $Z=\int \mathcal{D} \varphi e^{-F_{s t} / T}$. The complete expression for $\mathbf{J}$ in the linear response approximation can be obtained after averaging of $\ln Z$ over disorder (2) by using Eq.(1) for $F_{s t}$. One obtains

$$
J_{z}=\left\langle\left\langle\frac{e \hbar N_{s}^{(1)}}{2 m_{\|}} \frac{\partial \varphi_{\mathbf{j}}}{\partial z}-\frac{e^{2} N_{s}^{(1)} \xi_{\|}}{m_{\|} c} A_{z}\right\rangle\right\rangle
$$

for the longitudinal component of the current and

$$
\begin{gathered}
\mathbf{J}_{\perp}=\sum_{\mathbf{g}} \frac{2 e a_{\perp} \xi_{\|} N_{s}^{(1)}}{\hbar} \mathbf{g} E_{\mathbf{g}}\left\langle\left\langle\sin \left(\varphi_{\mathbf{j}}-\varphi_{\mathbf{j}+\mathbf{g}}\right)\right\rangle\right\rangle- \\
\sum_{\mathbf{g}} \frac{4 e^{2} a_{\perp}^{2} \xi_{\|} N_{s}^{(1)}}{\hbar^{2} c} \mathbf{g} E_{\mathbf{g}}\left\langle\left\langle\cos \left(\varphi_{\mathbf{j}}-\varphi_{\mathbf{j}+\mathbf{g}}\right)\right\rangle\right\rangle\left(\mathbf{g} \mathbf{A}_{\perp}\right)
\end{gathered}
$$

for the transverse component of the current.

For simplicity we present here only the diamagnetic contribution to the $i$-th $(i=\|, \perp)$ component of the current

$$
J_{i}^{d i a}(z, \mathbf{j})=-\frac{c}{4 \pi \lambda_{i}^{2}} A_{i}(z, \mathbf{j}),
$$

where the longitudinal $\left(\lambda_{\|}\right)$and the transverse component $\left(\lambda_{\perp}\right)$ of the penetration depth are obtained as

$$
\lambda_{\|}^{-2}=\frac{4 \pi e^{2} N_{s}^{(1)}(T)}{c^{2} m_{\|} a_{\perp}^{2}},
$$

and

$$
\lambda_{\perp}^{-2}=\frac{8 \pi e^{2} N_{s}^{(1)}(T) E_{\perp}}{c^{2} \hbar^{2}}\left\langle\left\langle\cos \left(\varphi_{\mathbf{j}}-\varphi_{\mathbf{j}+\mathbf{g}}\right)\right\rangle\right\rangle .
$$

While $\lambda_{\|}(T)$ diverges at $T=T_{c}^{(1)}$ due to pair breaking in the SC wires, $\lambda_{\perp}(T)$ diverges at the global SC transition temperature $T=T_{c}$, where the phase coherence between neighboring wires is destroyed. The temperature and the randomness dependencies of $\lambda_{\|}(T)$ and $\lambda_{\perp}(T)$ also strongly differ each other. The transverse component of the penetration depth is determined by the phase-phase correlator, revealing non-linear temperature dependence and discontinuous behavior at the critical 
disorder strength. Nevertheless the longitudinal component of the penetration depth is given by the conventional London expression and does not depend on the disorder strength. Randomness in the Josephson coupling shifts $T_{c}$ to lower temperatures and, therefore, the magnetic field parallel to the $\mathrm{SC}$ wires penetrates easier into the organic superconductor. On the other hand, the type of disorder considered in this article does not break the Cooper pairs, keeping thus the penetration of a perpendicular magnetic field into the SC wires unchanged.

\section{CONCLUSIONS}

In this paper we report disorder-effects on $T_{c}$ and on the diamagnetism of quasi-1D superconductors with random Josephson-couplings. Interplay of non-magnetic disorder with quantum phase fluctuations plays a central role for the superconductor normal-metal phase transitions in this class of quasi-1D superconductors. Recent experimental data found in Refs.9,10 are shown to be consistent with the present theory. Quantum criticality is controlled by two quantities, namely disorder strength and a dynamical parameter of phase fluctuations. The present model's quantum criticality signals the existence of a quantum critical phase between SC- and normal phase. Its nature deserves further investigation.

In our study we neglect the effects of non-linear excitations, which are a subject of current interest in low dimensional systems. Note that this topic was explicitly studied by us for quasi-2D Josephson coupled superconductors in Ref. 38. As we have shown in the previous section, the classical motion of the phase is described by a system of coupled sine-Gordon type non-linear equations (24), which contains non-linear dynamic excitations as well as static topological defects. The self-consistent phonon approximation allows us to calculate the phasephase correlator between two arbitrary points $\mathbf{r}=\{z, \mathbf{j}\}$ and $\mathbf{r}^{\prime}=\left\{z^{\prime}, \mathbf{j}+\mathbf{g}\right\}$

$$
\left\langle\left\langle\cos \left(\varphi_{\mathbf{j}}(z)-\varphi_{\mathbf{j}+\mathbf{g}}\left(z^{\prime}\right)\right)\right\rangle\right\rangle \equiv e^{-S_{\alpha}\left(z-z^{\prime}, \mathbf{g}, T\right)},
$$

which can be shown to decrease at $\mathbf{g} \rightarrow 0$ and $\left|z-z^{\prime}\right| \rightarrow$ $\infty$ as a power law $\sim\left(\xi_{\|} /\left|z-z^{\prime}\right|\right)^{\beta(T)}$, setting up a quasi-long-range order and implying the existence of a Berezinskii-Kosterlitz-Thouless (BKT) topological phase transition $^{45}$ (perhaps at $T=0$ ) in a single SC wire. The critical index $\beta(T)$ contains both phonon and vortex contributions. Although the phonon contribution to $\beta(T)$ can be calculated within the SCHA, it is not clear how the vortex contribution changes the former one. In our knowledge, the mechanism of excitations of the vortices with opposite fugacities and their binding in quasi-1D superconductors has not been adequately studied, and the topic needs further investigations.

\section{ACKNOWLEDGMENT}

This research was supported by the DFG under grant Op28/7-1. E.N. thanks D. Jerome for helpful communication. R.O. is grateful for hospitality and support extended to him by the IPhT at CEA Saclay.

\section{APPENDIX}

In order to calculate $S_{\alpha}(\mathbf{g}, 0)$ for $\mathbf{g}=\mathbf{e}_{x} N_{x}+\mathbf{e}_{y} N_{y}$ (where $\mathbf{e}_{x}, \mathbf{e}_{y}$ are unit vectors and $N_{x}, N_{y}$ are the number of unit cells in directions of $x, y$, correspondingly) we rewrite Eq.(34) in the following form:

$S_{\alpha}(\mathbf{g}, 0)=\pi \alpha \int_{-1}^{1} \frac{d q_{z}}{2 \pi} \int_{-\pi}^{\pi} \frac{d q_{x}}{2 \pi} \int_{-\pi}^{\pi} \frac{d q_{y}}{2 \pi} \frac{1-\cos \left(\mathbf{q}_{\perp} \mathbf{g}\right)}{\sqrt{q_{z}^{2}+\delta_{q u}^{2}(0) \omega_{\perp}^{2}}}$

where $\omega_{\perp}^{2}\left(q_{x}, q_{y}\right)=2\left(2-\cos q_{x}-\cos q_{y}\right)$ and $\mathbf{q}_{\perp}=$ $\left\{q_{x}, q_{y}\right\}$. Introducing a new variable $z=q_{z} / \delta_{q u}(0)$ and using the transformation

$$
\frac{1}{\sqrt{z^{2}+\omega_{\perp}^{2}}}=\frac{2}{\sqrt{\pi}} \int_{0}^{\infty} d t e^{-t^{2}\left(z^{2}+\omega_{\perp}^{2}\right)},
$$

one can integrate out $z, q_{x}$ and $q_{y}$ in Eq.(56). Finally, $S_{\alpha}(\mathbf{g}, 0)$ is expressed as an integral over $u=2 t^{2}$ as

$$
\begin{gathered}
S_{\alpha}(\mathbf{g}, 0)=\frac{\alpha}{2} \int_{0}^{\infty} \frac{d u}{u} e^{-2 u}\left[I_{0}^{2}(u)-I_{N_{x}}(u) I_{N_{y}}(u)\right] \times \\
\times \operatorname{erf}\left(\delta_{q u}^{-1}(0) \sqrt{u / 2}\right)
\end{gathered}
$$

where $I_{N}(u)$ is the Bessel function of an imaginary argument, and $\operatorname{erf}(z)=\frac{2}{\sqrt{\pi}} \int_{0}^{z} d t e^{-t^{2}}$ is the error function. By using the following asymptotic expressions for $I_{N}(u)$,

$$
I_{\nu}(z)= \begin{cases}\sum_{k=0}^{\infty} \frac{1}{k ! \Gamma(\nu+k+1)}\left(\frac{z}{2}\right)^{\nu+2 k}, & 0<z<\sqrt{\nu+1} \\ \frac{e^{z}}{\sqrt{2 \pi z}}\left[1-\frac{(\nu+1 / 2)(\nu-1 / 2)}{2 z}\right], & z>>\nu\end{cases}
$$

and for $\operatorname{erf}(z)$,

$$
\operatorname{erf}(z)= \begin{cases}\frac{2}{\sqrt{\pi}} \sum_{k=1}^{\infty}(-1)^{k+1} \frac{z^{2 k-1}}{(2 k-1)(k-1) !}, & z<1 \\ 1-\frac{e^{-z^{2}}}{\sqrt{\pi} z} \sum_{k=0}^{\infty}(-1)^{k} \frac{(2 k-1) ! !}{\left(2 x^{2}\right)^{k}}, & z>>1\end{cases}
$$

we get for, e.g. $S_{\alpha}(N, 0)$ at $N_{x}=N, N_{y}=0$ the following expression

$$
S_{\alpha}(N, 0)=\alpha \ln \frac{C_{1}}{\delta_{q u}(0)}-\frac{\alpha}{4 \pi} \frac{C_{2}}{N},
$$

i.e. $e^{-S_{\alpha}(N, 0)} \sim\left(\delta_{q u}(0)\right)^{\alpha} \exp \left(\frac{\alpha}{4 \pi} \frac{C_{2}}{N}\right)$, where $C_{1}$ and $C_{2}$ are constants of order of unity. If we take only the first terms in the expansions of $I_{N}(z)$ and $\operatorname{erf}(z)$, we get $C_{1}=1 / \sqrt{2}$ and $C_{2}=1$. Higher order contributions correct only these constants. Thus it is seen from Eq.(59) 
that the phase-phase correlator in the transverse direction saturates at $T=0$ to its asymptotic value of $\delta_{q u}^{\alpha}(0)$ for distances of the unit cell size $a_{\perp}$.

The correlator $S_{\alpha}(\mathbf{g}, T)$ at $T \neq 0$ is also calculated in the same way as $S_{\alpha}(\mathbf{g}, 0)$ was obtained above. Using in Eq.(40) the representation $\operatorname{coth}(\pi z)=\frac{z}{\pi} \sum_{n=-\infty}^{\infty} \frac{1}{n^{2}+z^{2}}$, the correlator $S_{\alpha}(\mathrm{g}, T)$ assumes the following form

$$
\begin{aligned}
& S_{\alpha}(\mathbf{g}, T)=\frac{T}{\pi \delta_{q u}(T) T_{c}^{(1)}} \sum_{n=-\infty}^{\infty} \int_{0}^{\frac{1}{\delta_{q u}}} d q_{z} \int_{-\pi}^{\pi} \frac{d q_{x}}{2 \pi} \times \\
& \times \int_{-\pi}^{\pi} \frac{d q_{y}}{2 \pi} \frac{1-\cos (\mathbf{q} \perp \mathbf{g})}{\left(\frac{T}{\alpha \delta_{q u}(T) T_{c}^{(1)}}\right)^{2} n^{2}+q_{z}^{2}+\omega_{\perp}^{2}(T)}
\end{aligned}
$$

We use the transformation $1 / a=\int_{0}^{\infty} e^{-a u} d u$ in Eq.(60) and carry out the integrations over $q_{z}, q_{x}$ and $q_{y}$. For the particular case of $N_{x}=N$ and $N_{y}=0$, the expression for $S_{\alpha}(\mathrm{g}, T)$ is reduced into the following form

$$
\begin{aligned}
& S_{\alpha}(N, T)=\frac{\alpha}{2 \sqrt{\pi}} \int_{0}^{\infty} \frac{d u}{\sqrt{u}} e^{-2 u} I_{0}(u)\left[I_{0}(u)-I_{N}(u)\right] \times \\
& \times \operatorname{erf}\left(\delta_{q u}^{-1}(T) \sqrt{u / 2}\right) \Phi\left(u, \frac{T}{\sqrt{2} \alpha \delta_{q u}(T) T_{c}^{(1)}}\right),
\end{aligned}
$$

where $\Phi(u, \tau)$ is given by

$$
\begin{aligned}
\Phi(u, \tau) & =\tau \sum_{n=-\infty}^{\infty} \exp \left\{-u \tau^{2} n^{2}\right\} \\
& =\left\{\begin{array}{ll}
\tau\left(1+2 e^{-u \tau^{2}}\right), & u>\tau^{-2} \\
\sqrt{\pi / u}+\tau, & u<\tau^{-2}
\end{array}\right\} ;
\end{aligned}
$$

here $\tau$ represents the normalized temperature (see Eq.(54)) and the sum in Eq.(62) is also known as the so-called EllipticTheta-function $\theta_{3}\left(0, \exp \left(-u \tau^{2}\right)\right),{ }^{46}$. Using the asymptotic expressions for the Bessel and the error function as well as for $\Phi(u, \tau)$ in Eq.(61), we get the following explicit expressions for $\exp \left\{-S_{\alpha}(N, T)\right\}$

$$
e^{-S_{\alpha}(N, T)}= \begin{cases}\delta_{q u}^{\alpha} \exp \left\{-C \frac{T}{\delta_{q u} T_{c}^{(1)}}+\frac{\alpha}{4 \pi} \frac{1}{N}\right\}, & 0 \leq 2 \delta_{q u}^{2}<1<N<\left(\frac{\sqrt{2} \alpha \delta_{q u} T_{c}^{(1)}}{T}\right)^{2} \\ \delta_{q u}^{\alpha} \exp \left\{-C \frac{T}{\delta_{q u} T_{c}^{(1)}}+\frac{C_{3}}{N^{1 / 2}} \frac{T}{\delta_{q u} T_{c}^{(1)}}\right\}, & 0 \leq 2 \delta_{q u}^{2}<1<\left(\frac{\sqrt{2} \alpha \delta_{q u} T_{c}^{(1)}}{T}\right)^{2}<N \\ \left(\frac{T}{\alpha T_{c}^{(1)}}\right)^{\alpha} \exp \left\{-C \frac{T}{\delta_{q u} T_{c}^{(1)}}+\frac{C_{4}}{N^{1 / 2}} \frac{T}{\delta_{q u} T_{c}^{(1)}}\right\}, & 0 \leq 2 \delta_{q u}^{2}<\left(\frac{\sqrt{2} \alpha \delta_{q u} T_{c}^{(1)}}{T}\right)^{2}<1<N \\ \exp \left\{-C \frac{T}{\delta_{q u} T_{c}^{(1)}}+\frac{C_{4}}{N^{1 / 2}} \frac{T}{\delta_{q u} T_{c}^{(1)}}\right\}, & 0 \leq\left(\frac{\sqrt{2} \alpha \delta_{q u} T_{c}^{(1)}}{T}\right)^{2}<2 \delta_{q u}^{2}<1<N\end{cases}
$$

where $C_{3}, C_{4}$, and $C_{5}$ are again constants of order unity.

1 D. Jérome, A. Mazaud, M. Ribault, and K. Bechgaard, J. Physique Lett. 41, L95 (1980).

2 C. Bourbonnais and D. Jérome, arXiv:0904.0617v1 [condmat.str.el] 3 Apr. 2009.

3 M. -Y. Choi, P. M. Chaikin, S. Z. Huang, P. Haen,E. M. Engler, and R. L. Greene, Phys. Rev. B 25, 6208 (1982).

4 S. Bouffard,M. Ribault, R. Brusetti, D. Jérome, and K. Bechgaard, J. Phys. C 15, 2951 (1982).

5 B. J. Powell, J. Phys.: Condens. Matter 20, 345234 (2008).

6 A. A. Abrikosov, J. Low Temp. Phys. 53, 359 (1983).

7 J. Shinagawa, Y. Kurosaki, F. Zhang et al., Phys. Rev. Lett. 98, 147002 (2007).

8 T. Ishiguro, K. Yamaji, and G. Saito, Organic Superconductors (Springer-Verlag, Heidelberg, 1998).

9 N. Joo, P. A.-Senzier, C. Pasquier, P. Monod, D. Jérome, and K. Bechgaard, J. Euro. Phys. B 40, 43 (2004).

10 N. Joo, P. Auban-Senzier, C. R. Pasquier, D. Jérome, and
K. Bechgaard, Europhys. Lett. 72, 645 (2005).

11 B. J. Powell and R. H. McKenzie, Phys. Rev. B 69, 024519 (2004).

12 P. W. Anderson, J. Phys. Chem. Solids 11, 26 (1959).

13 A. A. Abrikosov and L. P. Gor'kov, Zh.Eksp.Teor.Fiz. 39, 1781 (1960) [Sov.Phys.JETP 12, 1243 (1961)].

14 V. M. Galitski and A. I. Larkin, Phys. Rev. B 66, 064526 (2002).

15 C. C. Tsuei and J. R. Kirtley, Rev. Mod. Phys. 72, 969 (2000).

16 P. Lee, Phys.Rev. Lett. 71, 1887, (1993).

17 P. J. Hirschfeld and N. Goldenfeld, Phys. Rev. B 48, 4219 (1993).

18 Y. Suzumura and H. J. Schulz, Phys. Rev. B 39, 11398 (1989).

19 E. P. Nakhmedov and E. V. Tahirov, J. Phys.: Cond. matter 6, 2245 (1994); ibid 62259 (1994). 
20 E. P. Nakhmedov and M. Hüner, Physica C 299, 301 (1998)

21 M. Ma and P. A. Lee, Phys. Rev. B 32, 5658 (1985).

22 R. Oppermann, Physica A 167, 301 (1990).

23 A. Ghosal, M. Randeria, and N. Trivedi, Phys. Rev. B 65, 014501 (2001).

24 B. Spivak, A. Zyuzin, and M. Hruska, Phys. Rev. B 64, 132502 (2001).

25 V. M. Galitski and A. I. Larkin, Phys. Rev. Lett. 87, 087001 (2001).

26 S. Sachdev, P. Werner, and M. Troyer, Phys. Rev. Lett. 92, 237003 (2004).

27 B. Spivak, P. Oreto, and S. A. Kivelson, Phys. Rev. B 77, 214523 (2008).

28 A. D. Maestro, B. Rosenow, M. Müller, and S. Sachdev, Phys. Rev. Lett. 101, 035701 (2008).

29 F. Mondaini, T. Paiva, R. dos Santos, and R. T. Scallettar, Phys. Rev. B 78, 174519 (2008)

30 R. Oppermann, Z. Phys. B - Cond. Mat., 63, 33 (1986)

31 A. M. Finkel'stein, Pis'ma Zh. Eksp. Teor. Fiz. 45, 37 (1987) [JETP Lett. 45, 46 (1987)].

32 P. W. Anderson, K. A. Muttalib, and T. V. Ramakrishnan, Phys. Rev. B 28, 117 (1983).

${ }^{33}$ M. A. Skvortsov and M. V. Feigel'man, Phys. Rev. Lett. 95, 057002 (2005).

${ }^{34}$ K. B. Efetov and A. I. Larkin, Zh.Eksp.Teor.Fiz. 66, 2290 (1974) [Sov.Phys.JETP 39, 1129 (1975)].

${ }^{35} \mathrm{Yu}$. A. Firsov and G. Yu. Yashin, Zh.Eksp.Teor.Fiz. 72, 1450 (1977) [Sov.Phys.JETP 45, 761 (1977)].

36 H. J. Schulz and C. Bourbonnais, Phys. Rev. B 27, 5856 (1983).
37 V. J. Emery and S. A. Kivelson, Nature 374, 434 (1995); Phys. Rev. Lett. 74, 3253 (1995).

38 E. P. Nakhmedov and Yu. A. Firsov, Physica C 295, 150 (1998).

39 T. M. Rice, Phys. Rev.140, A1889 (1965).

40 The ground state of a non-interacting electron gas in an isolated $1 D$ wire or in a quasi-1D system with a nested Fermi surface is a Peierls dielectric. Suggestion of $n$ - subband in the cross-section of a pure single wire surves to prevent the Peierls transition in the system. Disorder in the quasi-1D system destroys also the perfect nesting, suppressing at the same time the Peierls transition. If isolated wires contain point-like impurities as well, the ground state becomes a dielectric phase due to Anderson localization, and the transition becomes superconducting-insulator phase transition. In our case, anion disorder in the organic superconductors may not modify the Fermi surface.

41 According to Eq.(33) $\left|A_{\mathbf{q}}\right|^{2} \propto(1 / N)$, this approximation is valid for $N \gg 1$. Strictly speaking, this harmonic approximation is valid for small temperatures when $\sum_{\mathbf{q}}\left|A_{\mathbf{q}}\right|^{2}\left\langle\hat{N}_{\mathbf{q}}\right\rangle<1$, which means that few phonons are excited in the system.

${ }^{42}$ V. L. Pokrovskii and G. V. Uimin, Zh. Eksp. Teor. Fiz. 65, 1691 (1973) [Sov. Phys. JETP 38, 847 (1973)].

43 A. I. Larkin, Pis'ma v Zh. Eksp.Teor.Fiz. 2, 205 (1965)[Sov. Phys. JETP Lett.2, 130 (1965)].

44 Q. Yuan, H.-Y. Chen, H. Won, S. Lee, K. Maki, P. Thalmeier, and C. S. Ting, Phys. Rev. B 68, 174510 (2003).

45 See, e.g. P. Minnhagen, Rev. Mod. Phys. 59, 1001 (1987).

46 Mathematica handbook. 